\title{
EMISIÓN DE METANO ENTÉRICO EN SISTEMAS PASTORILES: ESTRATEGIAS DE REDUCCIÓN CON POTENCIAL PRÁCTICO
}

\section{ENTERIC METHANE EMISSION IN PASTORAL SYSTEMS: REDUCTION STRATEGIES WITH PRACTICAL POTENTIAL}

\author{
Juan Vargas ${ }^{1}$ \\ ${ }^{1}$ Zootecnista, M.Sc. Producción Animal, Docente Facultad de Ciencias Pecuarias. U.D.C.A, calle 222 No. 55-37, Bogotá, \\ D.C., Colombia, e-mail: juan.dvargas@udca.edu.co
}

Rev. U.D.C.A Act. \& Div. Cient. 18(2): 417-424, Julio-Diciembre 2015

\section{RESUMEN}

La producción animal debe desarrollar sistemas más eficientes y menos perjudiciales con el ambiente. El metano entérico representa una pérdida energética para el rumiante y es un gas de efecto invernadero. Por lo tanto, el objetivo de esta revisión fue plantear estrategias, que permitan disminuir la producción de metano entérico, en sistemas pastoriles. Las características botánicas y el manejo de las pasturas modifican las emisiones de metano entérico. Es por ello, que la inclusión de leguminosas taníferas, el manejo eficiente de las pasturas y el aumento en la productividad animal constituyen herramientas, que habilitarían la disminución de la producción de metano por unidad de producto. Otras estrategias reportadas en la literatura deben ser investigadas con mayor detenimiento en sistemas pastoriles.

Palabras clave: Gases efecto invernadero, nutrición, rumiantes, sistemas pastoriles.

\section{SUMMARY}

Animal production must develop more efficient systems and less harmful with the environment. Enteric methane represents an energy loss for ruminant and it is a gas of greenhouse effect. Therefore, the objective of this review was to consider strategies that allow reduce enteric methane production in pastoral systems. The enteric methane emissions are modified by botanical characteristics and management. Therefore, the inclusion of tanniferous leguminous, efficient management of pastures and the increase in animal productivity are tools that allow decreasing methane production per unit of product. In pastoral systems other strategies reported in the literature should be investigated more closely.

Key words: Greenhouse gases, nutrition, ruminants, pastoral systems.

\section{INTRODUCCIÓN}

La producción animal enfrenta grandes retos hacia el futuro. $\mathrm{El}$ incremento en la demanda de alimentos, el crecimiento poblacional y la reducción de las áreas cultivables hace necesario aumentar la eficiencia animal. La FAO (2009) proyecta un incremento entre el 50 y 60\%, en relación al 2000, en la demanda de alimentos para el 2050, acompañado de una mayor preferencia por alimentos de origen animal. Este incremento es explicado por una alta tasa de natalidad y un mayor ingreso per cápita de países en desarrollo (Tilman et al. 2011).

Los recursos naturales que permitirían aumentar la producción de alimentos, generalmente, presentan limitaciones en forma, en calidad o en cantidad (Gerber et al. 2013). Algunos autores sugieren que ciertos países en desarrollo tendrían la capacidad de incrementar la producción animal; no obstante, estos lugares presentan claros indicios de deterioro ambiental (Steinfeld et al. 2006). Por otro lado, es reconocido que las actividades antropogénicas son responsables del desequilibrio en los ecosistemas naturales, registrándose un aumento en la temperatura atmosférica, incidencia de fenómenos extremos, deshielo de glaciares, acidificación de acuíferos, pérdida de biomasa, desertificación y disminución de las áreas de bosques (IPCC, 2013; Murgueitio et al. 2013).

El impacto ambiental, debido a la ganadería, es un tema de gran interés en la actualidad. Recientemente, se ha evaluado el impacto ambiental de los sistemas de producción animal, bajo enfoques integrales, como lo son el de huella de carbono, huella hídrica y ciclo de vida (Steinfeld et al. 2006; Hoekstra et al. 2011; Gerber et al. 2013); sin embargo, la complejidad en la comprensión holística de las interacciones entre los diferentes componentes de los sistemas ha resultado en la aceptación de asunciones y generalizaciones, que no reflejan las particularidades propias de cada sistema de 
producción (Gerber et al. 2013). Aunado a ello, es evidente el poco desarrollo investigativo en sistemas pastoriles en el trópico, lo que ha limitado la implementación de herramientas, que permitan disminuir las emisiones de gases de efecto invernadero (GEI), de rumiantes en esta zona (Vargas et al. 2012).

Entre los impactos ambientales de origen antropogénico que han sido estudiados con mayor detenimiento, se destacan la emisión de GEI. La quema de combustibles fósiles es la principal fuente de emisiones de dióxido de carbono, mientras que en la agricultura, se emite una mayor proporción de metano y de óxido nitroso, provenientes de la fermentación entérica y de la utilización de fertilizantes nitrogenados, respectivamente (Lascano et al. 2011).

La producción animal aporta el 14,5\% de las emisiones de GEI de origen antropogénico, de los cuales, el 20 y $41 \%$ provienen de los sistemas de producción bovina, destinados a leche y carne, respectivamente (Gerber et al. 2013). La emisión de metano entérico es la categoría con mayor participación en las emisiones de GEI, proveniente de la producción bovina, representando entre el 43 y 47\% (Gerber et al. 2013).

En Colombia, los sistemas de producción de rumiantes están soportados nutricionalmente en pasturas. La implementación de tecnologías adecuadas para las condiciones tropicales ha permitido un aumento en la producción bovina (Holmann et al. 2003); sin embargo, se considera que los parámetros productivos de la ganadería colombiana están muy lejos de países altamente competitivos (FEDEGAN, 2006). Esta menor productividad ha sido asociada con una mayor emisión de GEI, por unidad de producto (Gerber et al. 2013).

Finalmente, el metano es un gas con un potencial de calentamiento 28 veces mayor que el dióxido de carbono (IPCC, 2013) y su tiempo de vida en la atmósfera es de, aproximadamente, diez años (Moss et al. 2000); con ello, el metano representa una pérdida energética para el animal, que varía entre un 2 y $12 \%$ de la energía bruta consumida (Johnson \& Johnson, 1995; Kurihara et al. 1999). Lo anterior sugiere que el desarrollo de estrategias tendientes a disminuir las emisiones de metano entérico representaría una mayor productividad animal y una menor emisión de GEI del sector. El objetivo del presente documento fue revisar algunas estrategias, que permitan mitigar las emisiones de metano entérico, en sistemas pastoriles.

\section{MATERIALES Y MÉTODOS}

Se realizó una búsqueda sistémica de documentos, que evaluaran la producción de metano entérico de rumiantes, haciendo énfasis en sistemas pastoriles. Para tal fin, se utiliza- ron las bases de datos de Science Direct, Google Académico y SciELO. Adicionalmente, se emplearon las siguientes palabras y su combinación en idioma inglés y español: "methane enteric", "ruminants" y "pastorals systems". Los documentos fueron seleccionados por su relevancia. Algunos trabajos reportados en el documento fueron incluidos, para poder dar explicación a las variaciones en las emisiones de metano.

El objetivo de esta revisión es presentar estrategias, que permitan reducir las emisiones de metano entérico en sistemas pastoriles; esta revisión, se organizó de la siguiente forma: descripción de la formación de metano entérico en rumiantes y características de la pradera asociadas a la producción de metano entérico. Además, producto de esta revisión, se realizaron dos figuras que resumen los trabajos revisados.

\section{RESULTADOS Y DISCUSIÓN}

Debido a la diversidad de trabajos reportados en la literatura, este documento se enfoca en describir la producción e identificar características, que permitan explicar las variaciones en las emisiones de metano entérico, según la diversidad botánica y el manejo de la pradera y señalar estrategias, que permitan disminuir estas emisiones y establecer áreas con potencial de investigación. Por este motivo, se revisaron trabajos que hayan sido evaluados en modelos in vivo y que la emisión de metano se exprese por unidad de materia seca consumida, a menos que se especifique lo contrario en el texto.

\section{Formación de metano entérico}

El metano entérico es producto de la fermentación de macromoléculas en un medio anaerobio, resultado de un complejo sistema simbiótico, entre diferentes grupos microbiales, presentes a lo largo del tracto digestivo (Beauchemin et al. 2008). En el rumiante, el $90 \%$ de la producción de metano, tiene origen en el retículo-rumen y el $10 \%$ restante, se produce en el tracto digestivo posterior que, en su mayoría, es absorbido hacia la sangre, transportado a los pulmones y expulsado por la nariz, durante la respiración. El metano producido en el rumen es eliminado, a través de la boca y la nariz, por medio del eructo y la respiración (Murray et al. 1976; Lassey et al. 1997).

Los microorganismos del rumen metabolizan los carbohidratos de la dieta, convirtiéndolos, principalmente, en ácidos grasos volátiles, como acetato, propionato y butirato (Beauchemin et al. 2008). Además, procesos de biohidrogenación de ácidos grasos insaturados, de reducción de nitratos y de formación de ácido propiónico y metano son considerados sumideros de hidrógeno, lo que permite mantener un ambiente ruminal, capaz de desarrollar procesos eficientes de fermentación anaerobia (Russell \& Wallace, 1997). 
El metano es reducido, primordialmente, desde dióxido de carbono, por un grupo de microorganismos metanógenos del dominio Archaea, entre los que se destacan Methanobrevibacter ruminantium, Methanobacterium formicicum y Methanomicrobium mobile (Stewart et al. 1997; Attwood \& McSweeney, 2008). Los protozoarios, al parecer, tienen un papel preponderante en la producción de metano en el rumen, ya que adheridos a su pared se han observado microorganismos metanogénicos, sugiriendo una posible transferencia interespecífica de hidrógeno (Giuburunca et al. 2014).

Características de las praderas asociadas con la producción de metano entérico

La literatura reporta diversas estrategias, que permiten reducir las emisiones de metano entérico en rumiantes. Eckard et al. (2010) proponen que las estrategias de mitigación deben estar enfocadas en el manejo del hato y del animal, la modificación de las poblaciones ruminales y la optimización en la alimentación. En este mismo sentido, Vargas et al. (2012) sugieren que, en sistemas pastoriles, la modificación en las características de las pasturas permite modular las emisiones de metano entérico en rumiantes. El reconocimiento de las variaciones y el desarrollo de estrategias, que permitan disminuir las emisiones de metano entérico, son requisitos indispensables para desarrollar programas tendientes a reducir las emisiones de GEI del sector (Pickering et al. 2015)

\section{Diversidad botánica de la pradera}

La inclusión de diferentes cultivares de Lolium perenne con un mayor contenido de carbohidratos solubles ha sido asociada con una menor producción de metano (Jonker et al. 2014). La fermentación de carbohidratos solubles promueve la producción de propionato, compitiendo con la formación de metano (Moss et al. 2000). Ellis et al. (2012) simularon un incremento entre 20 y $90 \%$ en las concentraciones de carbohidratos solubles en la dieta de rumiantes y concluyeron que las emisiones de metano pueden ser incrementadas entre un 2 y $7 \%$, debido a una mayor fermentación ruminal; sin embargo, algunos autores sugieren que la inclusión de carbohidratos no estructurales en la dieta puede disminuir las emisiones de metano por unidad de producto, al incrementar la producción animal (Gerber et al. 2013).

Por otra parte, las especies C3 han sido asociadas con una menor producción de metano entérico. Archimède et al. (2011) reportan una reducción de $17 \%$ en las emisiones de metano de rumiantes alimentados con especies C3 respecto a C4; la menor producción de metano es explicada por la menor concentración de carbohidratos estructurales en especies C3 (Ellis et al. 2012). Asimismo, la literatura reporta una mayor producción de metano cuando se incluyen carbohidratos estructurales respecto a carbohidratos solubles, en la dieta de rumiantes (Moe \& Tyrrell, 1979).

Las emisiones de metano entérico, debido a la inclusión de leguminosas en la dieta de rumiantes, ha presentado gran variabilidad entre trabajos (Lee et al. 2004; Carulla et al. 2005). Esta variación puede ser explicada por el nivel de consumo, la digestibilidad de la dieta, la presencia de compuestos secundarios, entre otros (Hammond et al. 2014). Al respecto, Archimède et al. (2011) reportaron que las leguminosas tropicales producen $14 \%$ menos metano, que aquellas de zonas templadas; estos autores sugieren que la presencia de compuestos secundarios podría explicar esta menor producción. En la figura 1 es evidente que la inclusión de leguminosas taníferas en la dieta de rumiantes presenta una disminución en las emisiones de metano respecto a la inclusión de leguminosas, con bajos contenidos de taninos (Vargas et al. 2014).

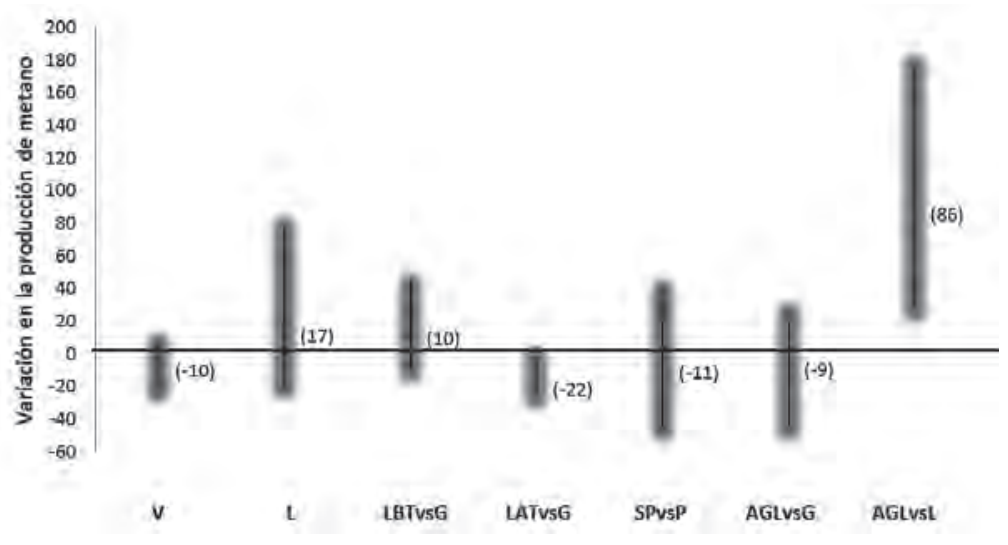

Figura 1. Variación (\%) de la emisión de metano entérico en rumiantes, debido a las características de las especies presentes en las praderas. V: Variedades de gramíneas; L: Leguminosas; LBT: Leguminosas bajas en taninos; LAT: Leguminosas altas en taninos; SP: Sistemas pastoriles; P: Pasturas; AGL: Asociación gramínea-leguminosa. () Valores entre paréntesis, promedio de trabajos revisados. 
Los taninos condensados en las dietas de rumiantes han sido asociados con la disminución en la degradación de la proteína (Waghorn, 2008), de la fibra (Tiemann et al. 2008a) y de la materia seca (Chipatecua et al. 2007). Algunos reportes mencionan un efecto bacteriostático o bactericida sobre poblaciones metanogénicas (Tavendale et al. 2005). La disminución en la fermentación de nutrientes del alimento y el efecto sobre poblaciones metanogénicas, se podría relacionar con una menor producción de metano entérico en rumiantes, alimentados con leguminosas taníferas (Tiemann et al. 2008b).

Pocos trabajos han evaluado las emisiones de metano cuando se incluyen arbóreas en la ración de rumiantes. Ramírez et al. (2010) reportan una disminución de $19 \%$ en las emisiones de metano por unidad de peso metabólico, cuando incluyó Salix spp., en la dieta de ovinos. A pesar de estos resultados es evidente la necesidad de evaluar la producción de metano en otros arreglos silvopastoriles.

\section{Manejo de la pradera}

Algunos trabajos sugieren que el estado de madurez de los forrajes no afecta las emisiones de metano entérico en rumiantes (Pinares et al. 2007; Molano \& Clark, 2008). La variación en la producción de metano podría ser explicada por las características de las especies evaluadas; por ejemplo, en la figura 2, se observa que la inclusión de gramíneas jóvenes en la dieta aumentó, ligeramente, las emisiones de metano, mientras que las de leguminosas, lo disminuye.
Los forrajes jóvenes presentan características que explicarían una menor producción de metano: la mayor concentración de proteína cruda (Lovett et al. 2004) y de ácidos grasos poli-insaturados y la menor concentración de carbohidratos estructurales (Aguilar et al. 2009). Aunque no es evidente la disminución en las emisiones de metano cuando se incluyen forrajes jóvenes, algunos autores sugieren que el aumento en la productividad animal resulta en una menor emisión de metano por unidad de producto (Clark et al. 2005; Pinares et al. 2007).

Por otro lado, la literatura reporta un disminución del $22 \%$ en las emisiones de metano por unidad de producto, cuando se utiliza un sistema de pastoreo rotacional respecto a uno continuo (DeRamus et al. 2003). La menor producción de metano en los sistemas rotacionales es explicada por una mayor capacidad de carga de sistema de producción resultado en una mayor producción por unidad de área. En este mismo sentido, McCaughey et al. (1997) mencionan que el aumento en la carga animal de 1,1 a 2,2 animales por hectárea, disminuyó las emisiones de metano en $15 \%$. La disminución en la concentración de carbohidratos estructurales en la pastura, debido a una modificación en el forraje remanente, aumenta la productividad animal y disminuye las emisiones de metano (Wims et al. 2010).

La fertilización de pasturas ha sido asociada con la disminución en la producción de metano, debido, posiblemente, a la mayor concentración de proteína y menor de carbohidratos en el forraje (Murray et al. 2001; Ellis et al. 2012; Purcell et

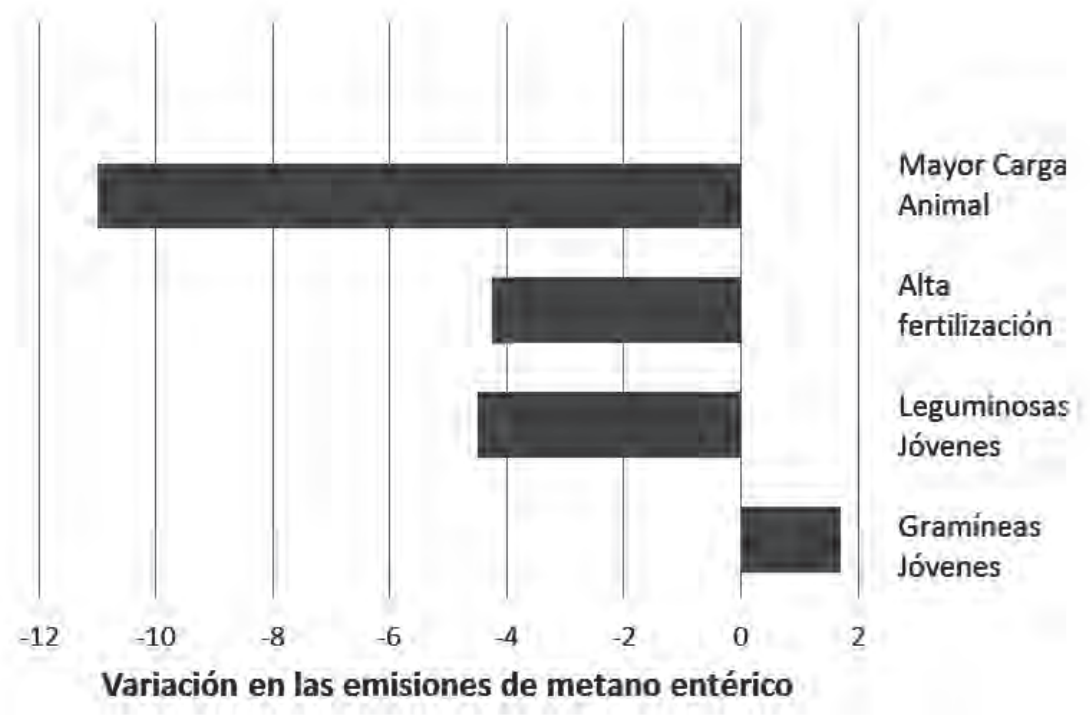

Figura 2. Variación (\%) de la emisión de metano entérico en rumiantes, debido al manejo de las praderas. 
al. 2014). Además, el aumento en la concentración de nitratos en forrajes fertilizados, se relaciona con la disminución en las emisiones de metano. Los nitratos se reducen hasta amonio en el rumen, pudiendo ser fuente de nitrógeno para algunas poblaciones de microorganismos, en especial, bacterias celulolíticas (Russell et al. 1992). Previamente, se ha sugerido que la inclusión de nitratos en la dieta de rumiantes, se relaciona inversamente con las emisiones de metano (Van Zijderveld et al. 2011) y se consideran una estrategia de suplementación en dietas, con bajos tenores de proteína (Leng, 2008).

\section{Conclusión}

La literatura reporta estrategias que permitirían disminuir hasta un $40 \%$ las emisiones de metano entérico de rumiantes, en sistemas pastoriles; sin embargo, existe gran variabilidad dentro de los diferentes trabajos revisados. La inclusión de leguminosas taníferas y la implementación de prácticas, que optimicen el uso de las pasturas, son estrategias promisorias, que permitirían disminuir las emisiones de metano y aumentar la productividad animal. La inclusión de arbóreas y el uso de especies o cultivares con mejor valor nutricional deben ser evaluados con mayor profundidad. Es evidente el poco desarrollo investigativo en regiones tropicales, resultando en una limitada implementación de prácticas de manejo adecuadas a las condiciones particulares de los sistemas de producción nacional.

Conflicto de intereses: El manuscrito fue preparado y revisado por el autor, quien declara que no existe conflicto de intereses que ponga en riesgo la validez de los resultados presentados.

\section{BIBLIOGRAFÍA}

1. AGUILAR, O.; MORENO, B.; CÁRDENAS, E.; PABÓN, M.; CARULLA, J. 2009. Efecto del consumo de kikuyo (Pennisetum clandestinum) o ryegrass (Lolium spp.) sobre la concentración de ácido linoléico conjugado y el perfil de ácidos grasos de la grasa láctea. Livestock research for rural development. Volume 21, Article \# 49. Disponible desde internet en: http:// www.lrrd.org/lrrd21/4/agui21049.htm (con acceso 23/01/2015).

2. ARCHIMÈDE, H.; EUGÈNE, M.; MARIE, C.; BOVAL, M.; MARTIN, C.; MORGAVI, D.P.; LECOMTE, P.; DOREAU, M. 2011. Comparison of methane production between C3 y C4 grass and legume. Animal Feed Science and Technology. 166-167: 54-64.

3. ATTWOOD, G.; MCSWEENEY, C. 2008. Methanogen genomics to discover targets for methane mitigation technologies and options for alternative $\mathrm{H}_{2}$ utilization in the rumen. Austral. J. Exp. Agr. 48:28-37.

4. BEAUCHEMIN, K.A.; KREUZER, M.; O'MARA, F.; MCALLISTER, T.A. 2008. Nutritional management for enteric methane abatement: a review. Austral. J. Exp. Agr. 48: 21-27.

5. CARULLA, J.E.; KREUZER, M.; MACHMÜLLER, A.; HESS, H.D. 2005. Supplementation of Acacia mearnsii tannin decreases methanogenesis and urinary nitrogen in forage-fed sheep. Austral. J. Agr. Res. 56:961-970.

6. CLARK, H.; PINARES-PATIÑO, C.; DEKLEIN, C. 2005. Methane and nitrous oxide emissions from grazed grasslands. En: McGilloway, D.A. (ed). Grassland: a global resource. Ed. Wageningen Academic Publishers. (Wageningen, Netherlands). p. 279-293.

7. CHIPATECUA, M.R.; PABÓN, M.L.; CÁRDENAS, E.A.; CARULLA, J.E. 2007. Efecto de la combinación de una leguminosa tanífera (Lotus uliginosus cv Maku) con Pennisetum clandestinum, sobre la degradación in vitro de proteína y materia seca. Rev. Col. Cienc. Pec. 20:40-48.

8. DERAMUS, H.A.; CLEMENT, T.C.; GIAMPOLA, D.D.; DICKISON, P.C. 2003. Methane emissions of beef cattle on forage: Efficiency of grazing management system. J. Environ. Quality. 32:269-277.

9. ECKARD, R.J.; GRAINGER, C.; DE KLEIN, C.A.M. 2010. Options for the abatement of methane and nitrous oxide from ruminant production: A review. Livestock Sci. 130:47-56.

10. ELLIS, J.L.; DIKSTRA, J.; FRANCE, J.; PARSONS, A.J.; EDWARDS, G.R.; RASMUSSEN, S.; KEBREAB, E.; BANNINK, A. 2012. Effect of high-sugar grasses on methane emissions simulated using a dynamic model. J. Dairy Sci. 95:272-285.

11. FAO. 2009. El estado mundial de la agricultura y la alimentación. Food and Agriculture Organization of the United Nations (FAO). (Roma, Italy). p.10-34. Disponible desde internet en: http://www.fao.org/publications/sofa/2013/es/ (con acceso 23/01/2015).

12. FEDEGAN. 2006. Plan estratégico de la ganadería Colombiana 2019. Federación colombiana de ganaderos (FEDEGAN). (Bogotá, Colombia). Disponible desde internet en: http://www.fedegan.org.co//plan- 
estrategico-de-la-ganaderia-colombiana-2019_(con acceso 23/01/2015).

13. GERBER, P.J.; STEINFELD, H.; HENDERSON, B.; MOTTET, A.; OPIO, C.; DIJKMAN, J.; FALCUCCI, A; TEMPIO, G. 2013. Tackling climate change through livestock - A global assessment of emissions and mitigation opportunities. Food and Agriculture Organization of the United Nations (FAO). (Roma, Italia). Disponible desde internet en: http://www. fao.org/docrep/018/i3437e/i3437e.pdf (con acceso 23/01/2015).

14. GIUBURUNCA, M.; CRISTE, A.; COCAN, D.; CONSTANTINESCU, R.; RADUCU, C.; MIRESAN, V. 2014. Methane production in the rumen and its influence on global warming. ProEnvironment. 7:64-70.

15. HAMMOND, K.J.; PACHECO, D.; BURKE, J.L.; KOOLAARD, J.P.; MUETZEL, S.; WAGHORN, G.C. 2014. The effects of fresh forages and feed intake levels on digesta kinetics and enteric methane emissions from sheep. Animal Feed Sci. Techn. 193:32-43.

16. HOEKSTRA, A.Y.; CHAPAGAIN, A.K.; ALDAYA, M.M.; MEKONNEN, M.M. 2011. The water footprint manual. Setting the global standard. Ed. Earthscan publishes. (London, UK). p.73-98. Disponible desde internet en: http://www.waterfootprint.org/?page=files/ WaterFootprintAssessmentManual (con acceso 23/01/2015).

17. HOLMANN, F.; RIVAS, L.; CARULLA, J.; RIVERA, B.; GIRALDO, L.; GUZMÁN, S.; MARTÍNEZ, M.; MEDINA, A.; FARROW, A. 2003. Evolución de los sistemas de producción de leche y su interrelación con los mercados: Un análisis del caso colombiano. Centro Internacional de Agricultura Tropical (CIAT), International Livestock Research Institute (ILRI) y Systemwide Livestock Program (SLP). Documento de trabajo \# 193. (Cali, Colombia). p.10-100.

18. IPCC. 2013. The final draft report-Technical Assessment. Work group I contribution to the IPCC 5th assessment report "Climate change 2013: The physical science basis". Stocker, T.F.; Qin, D.; Plattner, G.K.; Tignor, M.; Allen, S.K.; Boshung, J.; Nauels, A.; Xia, Y.; Bex, V.; Midgley, P.M. eds Disponible en internet en: http://www.ipcc.ch/report/ar5/wg1/ (con acceso 23/01/2015).

19. JOHNSON, K.A.; JOHNSON, D.E. 1995. Methane emissions from cattle. J. Animal Sci. 73:2483-2492.
20. JONKER, A.; MOLANO, G.; SANDOVAL, E.; TAYLOR, P.S.; ANTWI, C.; COSGROVE, G.P. 2014. Brief communication: Methane emissions by sheep offered high-sugar or conventional perennial ryegrass at two allowance. Proc. New Zealand Soc. Animal Prod. 74:145-147.

21. KURIHARA, M.; MAGNER, T.; HUNTER, R.A.; MCCRABB, G.J. 1999. Methane production and energy partition of cattle in the tropics. British J. Nutrition. 81:227-234.

22. LASCANO, C.E.; CARULLA, J.E.; VARGAS, J.J. 2011. Strategies for reducing methane emissions from ruminants. Rev. Bras. Geogr. Física. 06:1315-1335.

23. LASSEY, K.; MARCUS, J.; ULYATT, J.; MARTIN, R.; WALKER, C.; SHELTON, D. 1997. Methane emissions measured directly from grazing livestock in New Zealand. Atmosph. Environ. 31(18):2905-2914.

24. LEE, J.M.; WOODWARD, S.L.; WAGHORN, G.C.; CLARK, D.A. 2004. Methane emissions by dairy cows fed increasing proportions of with clover (Trifolium repens) in pastures. Proc. New Zealand Grassland Assoc. 66:151-155.

25. LENG, R.A. 2008. The potential of feeding nitrate to reduce enteric methane production in ruminants. A Report. The Department of Climate Change, Commonwealth Government of Australia. Disponible desde internet en: http://www.penambulbooks.com/ Downloads/Leng-Final\%20Modified\%20\%2017-92008.pdf (con acceso 23/01/2015).

26. LOVETT, D.K.; BORTOLOZZO, A.; CONAGHAN, P.; O'KIELY, P.O.; O'MARA, F.P. 2004. In vitro total and methane gas production as influenced by rate of nitrogen application, season of harvest and perennial ryegrass cultivar. Grass Forage Sci. 59:227-232.

27. MCCAUGHEY, W.P.; WITTENBERG, K.; CORRIGAN, D. 1997. Methane production by steers on pasture. Can. J. Animal Sci. 77:519-524.

28. MOE, P.W.; TYRRELL, H.F. 1979. Methane production in dairy cows. J. Dairy Sci. 62:1583-1586.

29. MOLANO, G.; CLARK, H. 2008. The effect of level of intake and forage quality on methane production by sheep. Austral. J. Exp. Agr. 48:219-222.

30. MOSS, A.; JOUANY, J.P.; NEWBOLD, J. 2000. Methane production by ruminants: its contribution to global warming. Ann. Zootechnie. 29:231-253. 
31. MURRAY, P.J.; GILL, E.; BALSDON, S.L.; JARVIS, S.C. 2001. A comparison of methane emissions from sheep grazing pastures with differing management intensities. Nutrient Cycling in Agrosystems. 60:9397.

32. MURRAY, R.M.; BRYANT, A.M.; LENG, R.A. 1976. Rates of production of methane in the rumen and large intestine of sheep. British of Nutrition. 36:1-14.

33. MURGUEITIO, E.; CHARA, J.D.; SOLARTE, A.J.; URIBE, F.; ZAPATA, C.; RIVERA J.E. 2013. Agroforestería pecuaria y sistemas silvopastoriles intensivos (SSPi) para la adaptación ganadera al cambio climático. Rev. Col. Cienc. Pec. 26:313-316.

34. PICKERING, N.K.; CHAGUNDA, M.G.G.; BANOS, G.; MRODE, R.; MCEVAN, J.C.; WALL, E. 2015. Genetic parameters for predicted methane production and laser methane detector measurements. J. Anim. Sci. 93:11-20.

35. PINARES, C.S.; WAGHORN, G.C.; MACHMÜLLER, A.; VLAMING, B.; MOLANO, G.; CAVANAGH, A.; CLARK, H. 2007. Methane emissions and digestive physiology of non-lactating dairy cows fed pasture forage. Can. J. Animal Sci. 86:601-613.

36. PURCELL, P.J.; BOLAND, T.M.; O'KIELY, P. 2014. The effect of water-soluble carbohydrate concentration and type on in vitro rumen methane output of perennial ryegrass determined using a 24-hour batchculture gas production technique. Irish J. Agr. Food Res. 53: 21-36.

37. RAMÍREZ, C.A.; BARRY, T.N.; MARRINER, A.; LÓPEZVILLALOBOS, N.; MCWILLIAM, E.L.; LASSEY, K.R.; CLARK, H. 2010. Effects of grazing willow fodder blocks upon methane production and blood composition in young sheep. Animal Feed Sci. Techn. 155:33-43.

38. RUSSELL, J.B.; O CONNOR, J.D.; FOX, D.G.; VAN SOEST, P.J.; SNIFFEN, C.J. 1992. A net carbohydrate and protein system for evaluating cattle diets: I. Ruminal fermentation. J. Animal Sci. 70:3551-3561.

39. RUSSELL, J.B.; WALLACE, R.J. 1997. Energy-yielding and energy-consuming reactions. En: Hobson, P.N.; Stewart, CS. (eds). The rumen microbial ecosystem. Ed. Blackie Academic \& Professional Editorial. Second Edition. (London, UK). p.246-279.
40. STEINFELD, H.; GERBER, P.; WASSENAAR, T.; CASTEL, V.; ROSALES, M.; DE HAAN, C. 2006. Livestock's long shadow - Environmental issues and options. Food and Agriculture Organization of the United Nations (FAO). (Rome, Italy). Disponible desde internet en: http://www.fao.org/docrep/010/a0701e/ a0701e00.HTM (con acceso: 23/01/2015).

41. STEWART, C.S.; FLINT, H.J.; BRYANT, M.P. 1997. The rumen bacteria. En: Hobson, P.N.; Stewart, CS. (eds). The rumen microbial ecosystem. Ed. Blackie Academic \& Professional Editorial. Second Edition. (London, UK). p.10- 72.

42. TAVENDALE, M.H.; MEAGHER, L.; PACHECO, D.; WALKER, N.; ATTWOOD, G.T.; SIVAKUMARAN, S. 2005. Methane production from in vitro rumen incubations with Lotus pedunculatus and Medicago sativa, and effects of extractable condensed tannin fractions on methanogenesis. Animal Feed Sci. Techn. 123-124:403-419.

43. TIEMANN, T.T.; RAMÍREZ, G.; LASCANO, C.E.; KREUZER, M.; HESS, H.D. 2008a. The ruminal degradability of fibre explains part of the low nutritional value and reduce methanogenesis in highly tanniniferus tropical legumes. J. Sci. Food Agr. 88:1794-1803.

44. TIEMANN, T.T.; LASCANO, C.E.; WETTSTEIN, H.R.; MAYER, A.C.; KREUZER, M.; HESS, H.D. 2008b. Effect of the tropical tannin-rich shrub legumes Calliandra calothyrsus and Flemingia macrophylla on methane emission, nitrogen and energy balance in growing lambs. Animal. 2(5):790-799.

45. TILMAN, D.; BALZERB, C.; HILL, J.; BEFORTA, B.L. 2011. Global food demand and the sustainable intensification of agriculture. Proc. Nal Acad. Sci. 108:20260-20264.

46. VAN ZIJDERVELD, S.M.; GERRITS, W.J.J.; DIJKSTRA, J.; NEWBOLD, J.R.; HULSHOF, R.B.A.; PERDOK, H.B. 2011. Persistency of methane mitigation by dietary nitrate supplementation in dairy cows. J. Dairy Sci. 94:4028-4038.

47. VARGAS, J.; PABÓN, M.; CARULLA, J. 2014. Producción de metano in vitro en mezcla de gramíneas - leguminosas del trópico alto colombiano. Arch. Zoot. 63 (243):397-407.

48. VARGAS, J.; CÁRDENAS, E.; PABÓN, M.; CARULLA, J. 2012. Emisión de metano entérico en rumiantes en pastoreo. Arch. Zoot. 61(R):51-66. 
49. WAGHORN, G. 2008. Beneficial and detrimental effects of dietary condensed tannins for sustainable sheep and goat production-Progress and challenges. Animal Feed Sci. Techn. 147:116-139. ge mass on methane production, dry matter intake and milk production of grazing dairy cows during the mid-lactation period. J. Dairy Sci. 93(10):49764985.

50. WIMS, C.M.; DEIGHTON, M.H.; LEWIS, E.; O’LOUGHLiN, B.; DELABY, L.; BOLAND, T.M.; Recibido: Enero 29 de 2015

O’DONOVAN, M. 2010. Effect of pregrazing herba- Aceptado: Junio: 8 de 2015

\section{Cómo citar:}

Vargas, J. 2015. Emisión de metano entérico en sistemas pastoriles: estrategias de reducción con potencial práctico. Rev. U.D.C.A Act. \& Div. Cient. 18(2): 417- 424. 